\title{
Major Thought Restructuring: The Roles of Different Prefrontal Cortical Regions
}

\author{
Shima Seyed-Allaei ${ }^{1,2}$, Zahra Nasiri Avanaki ${ }^{3}$, Bahador Bahrami ${ }^{4}$, and Tim Shallice ${ }^{4,5}$
}

\begin{abstract}
An important question for understanding the neural basis of problem solving is whether the regions of human prefrontal cortices play qualitatively different roles in the major cognitive restructuring required to solve difficult problems. However, investigating this question using neuroimaging faces a major dilemma: either the problems do not require major cognitive restructuring, or if they do, the restructuring typically happens once, rendering repeated measurements of the critical mental process impossible. To circumvent these problems, young adult participants were challenged with a one-dimensional Subtraction (or Nim) problem [Bouton, C. L. Nim, a game with a complete mathematical theory. The Annals of Mathematics, 3, 35-39,
\end{abstract}

\section{INTRODUCTION}

Within human experimental psychology the effective study of problem solving goes back to Selz (1922) and the Gestalt School. However, its cognitive neuroscience is relatively new. An outstanding question is whether the left and right prefrontal cortices play qualitatively different roles in problem solving. On this issue, Goel (1995) distinguished between making a "vertical transformation" and a "lateral transformation" in problem solving. A vertical transformation is a move in a problem state space to a more detailed version of the same representation. By contrast Goel held a lateral transformation to be a move to a new problem state space. Thus, lateral transformation involves viewing the problem in new ways (see Figure 1).

Goel and colleagues hypothesized that vertical transformations involve the left $\mathrm{pFC}$ and lateral ones the right (see, e.g., Goel \& Grafman, 2000). They conducted a series of studies to test this hypothesis. In one fMRI study, Goel and Vartanian (2005) tested whether the right pFC activated in tackling problems considered to require lateral transformations. They used a version of the so-called Matchstick Problems (see Figure 1) developed by Guilford (1967) to measure divergent thinking. In the experimental condition, a 22-match formation of eight fully formed squares was presented to the participant. On

\footnotetext{
${ }^{1}$ University of Tehran, ${ }^{2}$ Institute for Research in Fundamental Sciences (IPM), Tehran, Iran, ${ }^{3}$ Shahid Beheshti University of Medical Sciences, Tehran, Iran, ${ }^{4}$ University College London, ${ }^{5}$ International School for Advanced Studies (SISSA), Trieste, Italy
}

1901] that can be tackled using two possible strategies. One, often used initially, is effortful, slow, and error-prone, whereas the abstract solution, once achieved, is easier, quicker, and more accurate. Behaviorally, success was strongly correlated with sex. Using voxel-based morphometry analysis controlling for sex, we found that participants who found the more abstract strategy (i.e., Solvers) had more gray matter volume in the anterior medial, ventrolateral prefrontal, and parietal cortices compared with those who never switched from the initial effortful strategy (i.e., Explorers). Removing the sex covariate showed higher gray matter volume in Solvers (vs. Explorers) in the right ventrolateral prefrontal and left parietal cortex.

each trial, the task was to remove a given number of matches to leave a specified number of whole squares. In a baseline condition, a set of matches had already been crossed out and the participant decided whether removal of those specified set of matches satisfied the trial goal. The experimental and baseline conditions clearly differed in difficulty, so a difficulty rating was developed and used as a covariate in the analysis. A conjunction analysis was employed to determine which regions were more activated both for solving Matchstick Problems versus baseline and for successful Matchstick Problems versus unsuccessful ones. These regions were the right ventrolateral $\mathrm{pFC}$ (BA 47) and, at weaker significance levels, the left middle frontal gyrus (BA 9) and the left frontal pole (BA 10).

It was argued that, of these regions, the right ventrolateral pFC was especially critical for lateral transformations, as a neuropsychological study of another version of the Matchstick task-that of Miller and Tippett (1996) - had found that patients with right frontal lesions, particularly right ventrolateral ones, but not those with left frontal lesions, were impaired on the Matchstick Problems. Moreover, of the 20 problems each patient had to tackle, solving five were held to involve a major shift in strategy. The patients with right frontal lesions and also right central-parietal lesions were significantly inferior to normal controls on these major set shift problems. Consequently, Goel and Vartanian (2005) concluded that the integrity of right BA 47 was necessary for the lateral transformations involved in Matchstick Problems. 
Figure 1. An example of a Matchstick problem and the difference between lateral and vertical transformation.

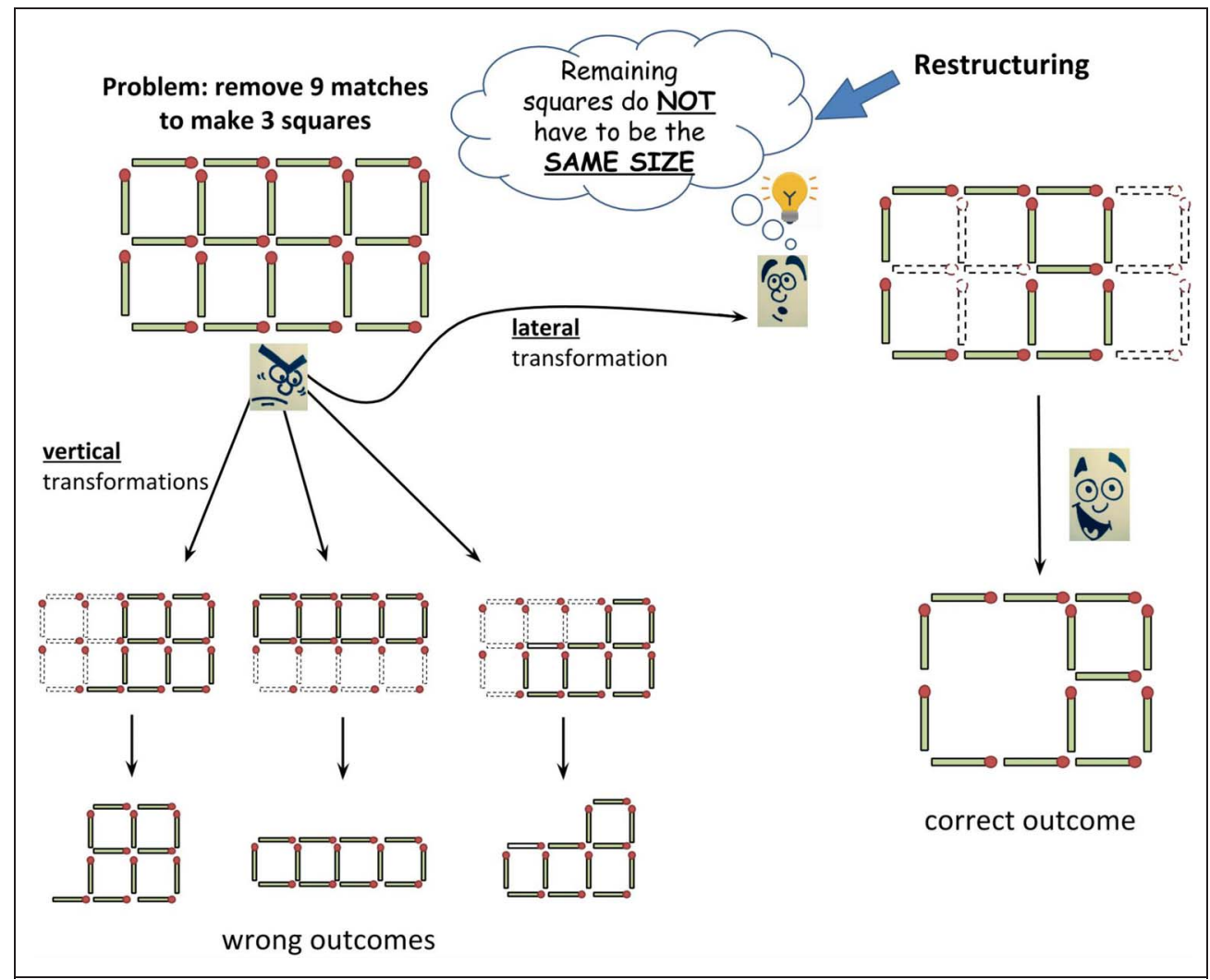

However, this thesis faces three difficulties. First, it is intuitively highly plausible that some Matchstick Problems do involve lateral transformations, as their use in studies of divergent thinking indicates. However, although Goel and Vartanian (2005) refer to support from unpublished pilot studies, they provided no formal empirical evidence that Matchstick Problems actually do involve lateral transformations (see Lee \& Johnson-Laird, 2013, for an empirical analysis of Matchstick Problems using an alternative conceptual approach). Moreover, as each participant tackles 20 experimental and 20 baseline problems in Goel and Vartanian's study, one does not know how many trials involve a new lateral transformation. Second, although Miller and Tippett's (1996) right frontal patients were indeed impaired on the major set shift problems, they were also impaired, although to a lesser extent, on nonmajor set shift problems, suggesting that some other aspect of the task may also be relevant. One possibility could concern the spatial processing since square detection loads on that type of operation. However, this possibility is somewhat reduced as Vartanian and Goel (2005) have also found BA 47 to be activated in an anagram solution task, which does not have such a clear spatial component.

Most critically, it is not even obvious what exactly a lateral transformation is. In the Goel and Vartanian (2005) study, lateral transformations are identified with Guilford's (1967) "set shifts" to overcome a cognitive fixation (see Figure 1). However, simple set shifting, as in paradigms like task switching, tends to involve more superior structures than BA 47 (Derrfuss, Brass, Neumann, \& von Cramon, 2005). Moreover, conceptually, as Goel and Vartanian point out, something more than simple set shifting appears to be required when solving difficult Matchstick Problems. Some implicit constraint in the way the participant tackles a task has to be removed (see Figure 1). So Matchstick Problems are often viewed as insight problems with their solution involving two stages (Knoblich, Ohlsson, \& Raney, 2001). There is an initial stage, in which a state of impasse occurs "due to a high probability of triggering an initial mental representation that has a low probability of leading to the solution" (Knoblich et al., 2001, p. 1001). This stage is depicted in Figure 1 by the several inadequate solutions in the lower left corner. This is eventually followed by what is typically a rapid process of reorganization of the problem space (e.g., the realization that "remaining squares do not have to be the same size"). According to Knoblich et al., the second stage occurs through the relaxation of inappropriate constraints, but Goel (2009) sees a more active process as necessary when claiming that the right $\mathrm{pFC}$ is critical in generating novel possibilities. This type of major set shift is now often called "restructuring" (e.g., Sandkühler \& Bhattacharya, 2008; Ohlsson, 1992) and corresponds to Goel's "lateral transformations."

How, though, does one assess whether a major restructuring process has occurred? This is necessary as, if one uses a set of problems of the same general type, once a solution is achieved, any similar problem, from 
then on, would not require the same type of, what we will call, major problem restructuring. It would merely require a minor adaptation of the achieved procedure. Thus, once a problem has been solved, encountering subsequent problems of the same nature with the restructured mental representations setup will trigger the appropriate solution.

One possibility for recognizing and identifying a moment of restructuring is to use self-reports. Sandkühler and Bhattacharya (2008) did this in an EEG study of the so called remote association problems (e.g., which word goes with "back, clip, wall"? Answer: "paper"), which only have a limited degree of complexity; we term such designs small-scale verbal restructuring. However, when one is solved, this does not greatly help in solving the next. These authors found that there was a strong trend for reduced EEG alpha power over the right frontal lobe just before production of solutions that were reported to have required restructuring. But there were no frontal effects related to ratings of the impasse state or the rated rapidity of solutions. However, source analysis was not used, so one cannot be certain that the critical process involved was indeed located in the right frontal lobe. For more discussion of small-scale verbal restructuring, see Kounios and Beeman (2014).

A summary of the existing literature indicates that major problem restructuring is nontrivial to study and that appropriate tasks have rarely been used. In particular, the key hypothesis derived from the work of Goel's group on whether right ventrolateral $\mathrm{pFC}$ has a key role in problem restructuring remains to be tested.

One way to study major problem restructuring specifically would be to use a very small number of qualitatively different problems, indeed ideally only one, in which the two-stage process discussed by Knoblich et al. (2001) reliably and observably occurs across a good percentage of participants. In a recent study, Seyed-Allaei, Amati, and Shallice (2010) examined a variant of the two-player game called Nim or the Subtraction Game (Bouton, 1901) and showed that this game had the desired properties (see also Hawes, Vostroknutov, \& Rustichini, 2012).

Traditionally, Nim or the Subtraction Game was played with heaps of stones. Each player could take any number of stones up to a given maximum. The player to take the last stone won. In the current version, in the Subtraction Game $G(n, k)$, which is mathematically isomorphic to that involving heaps of stones but strategically more transparent, players take turns in moving a pawn along a linear track of total length $n$ squares. On each turn, a player must move, but only by up to a maximum number, $k$, of squares (see Figure 2). In the $G(15,3)$ example in Figure 2, the first player starts by choosing from among the first three squares $(1,2,3)$ and lands on 2 . The next
Figure 2. An example game of $G(15,3)$ is shown on the left with the solution on the right. The critical positions for winning the $G(15,3)$ game are 3,7 , and 11 . Because the participant starts the game, if they figure out this strategy (first occupying 3, then 7 and then 11), they will always win.

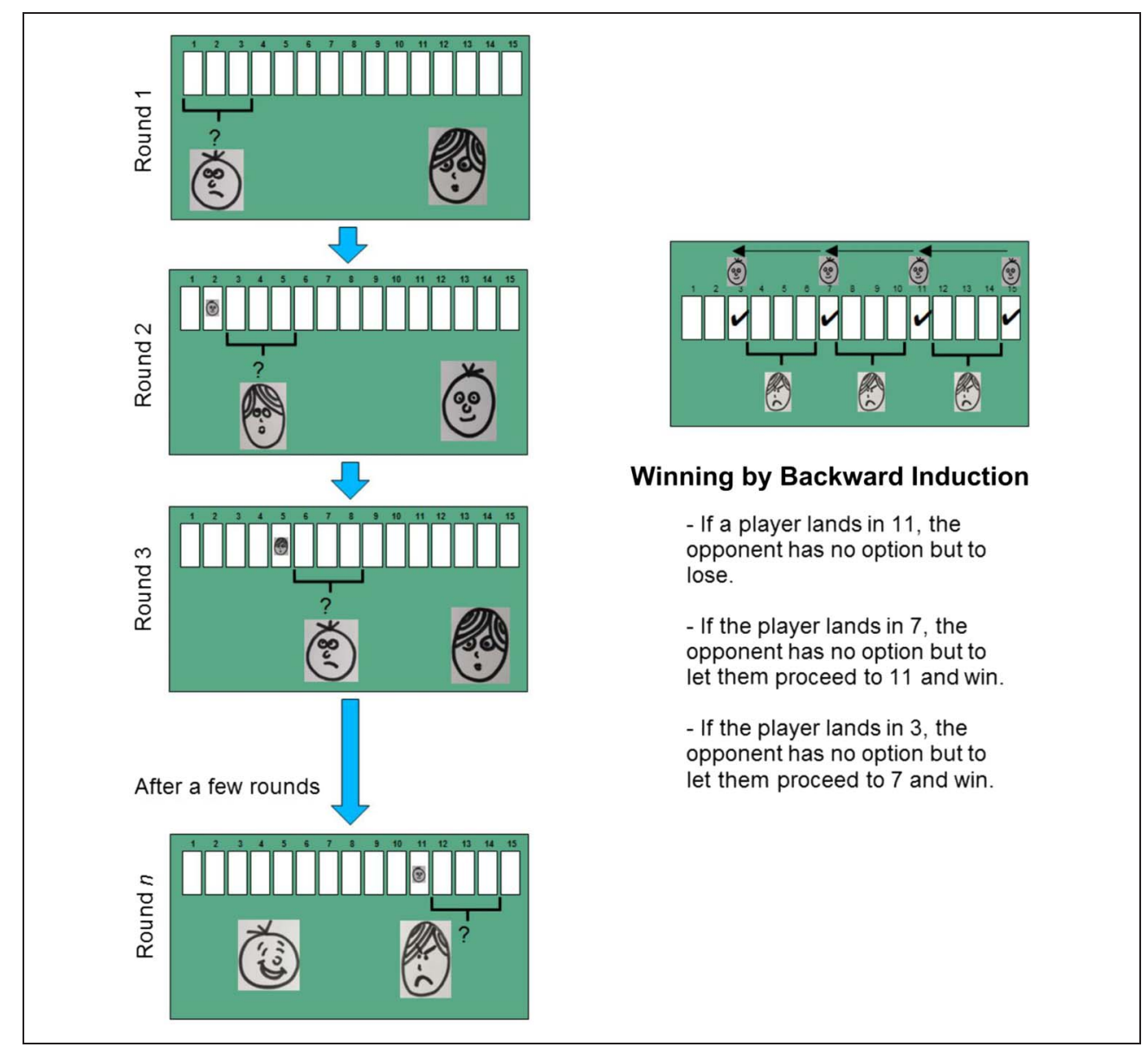


player chooses one from the next three squares $(3,4$, and 5 ) and lands on 5 . The winner is the player who moves the pawn onto the last square of the track (square 15) first. Seyed-Allaei et al. (2010) had participants play the Subtraction Game, not against another human opponent, but against a computer. Participants made the first move and so could always win if they used the correct strategy.

In the initial trials, though, most participants started searching the problem state space by trying to calculate forward toward their eventual goal. However, although the state space is well defined, it is far too large to be explored exhaustively. Many participants, however, gradually realized that certain squares (for $G(15,3)$, these would be 3,7, and 11) were better than others and tried to land on them. After playing a number of rounds of the game using such working forward procedures, some of these participants realized that a simple arithmetic procedure which involves working backward from the final square (see Figure 2, right column) can be used to determine the critical squares on which they should attempt to land. The participants who came to this much more efficient solution were called "Solvers" in contrast with "Explorers" who kept attempting just to work forward from the starting point. The difference between Solvers and Explorers is the strategy they use to win. Explorers are a mixed group also, including participants that have vaguely realized the importance of some or all of the critical positions through trial and error, but they did not know why those positions lead to a win.

The transition from intermittent success to continuously perfect performance in the typical Solver participant was shown by Seyed-Allaei et al. (2010) in three ways. Average RT for moves dropped and remained consistently low. In addition, the trajectory of mouse movements used on the trials during transition showed backward calculation. Moreover, participants' ability to verbalize their reasoning for the solution procedure at the end of the experiment indicated the conscious, rule-based nature of the successful strategy. We even observed a sudden "Aha!" reaction in some Solvers in their recorded voice, which was highly predictive of the change in strategy. Therefore, we believe that it is possible to determine the switch trial, if a participant is a Solver, reliably by examining the RT decline and performance surge.

Thus, this task permits a distinction between before and after the moment of problem restructuring, which could be used to identify its neural substrates. But such a single trial approach would be impractical with most neuropsychological and neuroimaging methodologies. Too many patients with lesions in the relevant area would be required for a neuropsychological assessment with reasonable power. It is also impractical to study major problem restructuring by conventional neuroimaging methods, for these methods require multiple repetitions of critical trials per participant and averaging across participants to segregate the signal from noise in the imaging data.
In this study, we have taken another approach. Instead of averaging across numerous participants to filter out the interindividual differences in behavior and brain, we chose to utilize the extensive individual variations that were previously observed in the Subtraction Game. Importantly, Seyed-Allaei et al. (2010) reported that $42 \%$ of their participants who played the Subtraction Game never discovered the backward solution and those who did showed great variation in how many trials they needed to find it. The restructuring occurred, on average, after the participant had played 19.2 rounds, but with individuals spanning a wide range from 3 to 28 rounds. Given the considerable degree of variation that exists in the local brain neuroanatomy of healthy normal adults, we asked whether the interindividual differences observed in problem restructuring (Seyed-Allaei et al., 2010) could be indicative of systematic variations in the local brain structure in human pFC. This method has now been used successfully to address a wide range of questions in cognitive neuroscience (e.g., Kanai, Bahrami, \& Rees, 2010; Mechelli et al., 2005; Maguire et al., 2000). Specifically, we hypothesized that successful changes of strategy would be associated with local regional differences in gray and/or white matter in pFC. We tested this hypothesis by using voxel-based morphometry to compare the problem Solvers (i.e., those who successfully restructured the problem to arrive at the optimal solution) with problem Explorers (who kept trying out heuristics without ever arriving at the ideal solution).

\section{METHODS \\ Participants}

Seventy-six healthy adults ( 41 women, mean age = 22.61 years, $S D=2.23$ years) participated in the study. The participants were all medical students at Shahid Beheshti University and University of Tehran. The experiment was approved by the local ethics committee at the Institute for Research in Fundamental Sciences.

\section{Behavioral Task and Analysis}

We used the Subtraction Game, also known as the onedimensional Nim game $G(n, k)$, a two-player game with an optimal strategy, formalized by Bouton (1901). On each trial (Figure 2), the players take turns in moving a pawn by up to $k$ squares in a linear array of $n$ squares. The player that moves into the $n$th square wins the game. In Figure 2, the number of maximum move or $k$ is 3 , and the number of positions or $n$ is 15 . If the player who starts the game knows the winning strategy for the game's $n$ and $k$, they will always win and the player going second has no way to stop player 1 .

The winning strategy involves identifying the critical squares by backward induction that guarantees winning (Figure 2): To guarantee that one will be able to move to square $n$ on the $i$ th move $\left(\right.$ pos $\left._{i}=n\right)$, one should 
aim to arrive at square $p s_{i-1}=n-1-k$ on the preceding $(i-1)$ th move. From that square one's opponent can only move to $n-k, n-k+1, \ldots, n-1$, thus enabling one to reach square $n$ on the following move and so win the game. Thus, pos $_{i-1}$ is a critical (winning) square. Back propagation of the same procedure shows that an equivalent situation is present for $\operatorname{pos}_{i-2}=n-$ $2(k+1)$ and so on. In this way, one can identify the set of critical squares $\operatorname{pos}_{1}, \operatorname{pos}_{2}, \ldots$, pos $_{\mathrm{i}}$, where $0 \leq \operatorname{pos}_{1} \leq k$. If $n$ is divisible by $k+1$, then $p o s_{1}=0$ and the second player can always win (by moving to $\operatorname{pos}_{2}=k+1$ whatever the initial move of the first player); by contrast if $1 \leq$ $\operatorname{pos}_{1} \leq k$, the player who starts can move through a series of winning squares and so win the game in $i$ moves.

Participants played the game against the computer, starting the game on all trials. As in Seyed-Allaei et al. (2010), participants played 15 trials of $G(15,3)$ followed by 15 trials of $G(17,4)$. In $G(15,3)$, the player who reaches position 15 first wins the game (see Figure 2). Every player can move 1, 2, or 3 positions forward in one turn. Therefore, if player $\mathrm{A}$ is on square 11, the opponent can only go as far as 12,13 , or 14 , all of which would let player A reach position 15 in her next move. Therefore, player A's goal to reach 15 translates into landing in 11 , that is, $15-(3+1)$. By backward induction, to reach position 11, player $\mathrm{A}$ has to land in position 7 and before that in position 3. Following this strategy, as long as player A starts the game and lands in position 3, there is no way she could lose (Figure 2 ). Each $G(n, k)$ has a set of such critical positions that ensure the starting player's victory; for $G(15,3)$ they are $(3,7,11)$, and for $G(17,4)$ they are $(2,7,12)$.

The computer always played perfectly: If at any point in the game, the participant did not occupy the critical square available to them, then the computer would immediately occupy it, and from then on, the participant could not win the game. For example, in $G(15,3)$, if the participant did not start by occupying square 3 but instead moved to 2 , then the computer would seize the opportunity and move to square 3, and from there proceed to win the game. This ensured that participants could not win the game by random guessing, but only if they knew exactly what position to choose. In addition, this extremely opportunistic virtual opponent can serve to familiarize the participants with the concept of critical positions (Seyed-Allaei et al., 2010).

To categorize the participants as Solvers versus Explorers, a questionnaire was administered at the end of experiment. The participants were asked to write down the strategy they used in both games and also what they would use in a third game $G(19,5)$. An example of written answer referring to the second game, from an Explorer is: "I started from low numbers like 1 or 2 . Then from 7 I could go to 12 and win." A Solver, on the other hand, had written: "First I tried to understand the pattern of computer moves. Then I realized it is not necessary. After losing 6 times in the second game I realized there are critical positions. These positions were $4+1$ apart. Thus to always win we should occupy $17-5=12$, $12-5=7$ and $7-5=2$." This answer was followed by the correct solution to $G(19,5)$. The explicit reference to critical positions in the answer to the questionnaire was the key to categorizing participants as Solver versus Explorer. Those who performed correctly on at least the last trial and solved $G(19,5)$ correctly were categorized as Solvers and the others as Explorers. Protocols were assessed independently by two authors before the MRI analysis. There was a 100\% agreement between the raters in categorizing participants as Solvers or Explorers.

Within Solvers, the specific moment of strategy change (the switch trial) was defined as the trial after which all subsequent moves were correct and their average RT within the same game were less than 6 sec. This criterion was adopted from Seyed-Allaei et al. (2010), where two independent judges also examined the mouse movements and the recorded voice to identify the switch trial. Seyed-Allaei et al. (2010) showed that the conclusions from judges and debriefings during and after the games were consistent with RT decline and flawless performance after the switch.

\section{Data Acquisition and Preprocessing}

High-resolution anatomical images of the whole brain were acquired using 1.5 T MAGNETOM Avanto scanner (Siemens, Erlangen, Germany; 160 axial slice, $1 \mathrm{~mm}$ thick, echo time $=3.5 \mathrm{msec}$, repetition time $=12 \mathrm{msec}$ ). The images were processed using DARTEL (Ashburner, 2007). First, the images were segmented for gray matter and white matter via a rigid body. Then the nonlinear deformations for warping the images were determined. The images were then smoothed with a Gaussian kernel (FWHM $=12 \mathrm{~mm}$ ) and normalized to MNI space with preserving the amount of voxels (modulated). The resulting images represent the regional volume of gray matter and white matter.

\section{Voxel-based Morphometry Analysis}

We performed statistical analyses on local regional differences in gray and white matter volume by fitting a general linear model to data using Statistical Parametric Mapping (SPM8, www.fil.ion.ucl.ac.uk/spm). In the first analysis, a $t$ test was performed to compare Solvers versus Explorers gray and white matter volumes. In the second analysis, we introduced covariates of no interest for sex using ANCOVA. We followed these up by additional inclusion of participants' accuracy and mathematical ability as covariates of no interest. Accuracy was defined as the grand-averaged accuracy before switch moment. Consequently, for Explorers, accuracy was calculated over all trials (because they never reached the switch moment), whereas for Solvers, accuracy was calculated over the trials preceding the switch. Mathematical ability was assessed by collecting the participants' grades 


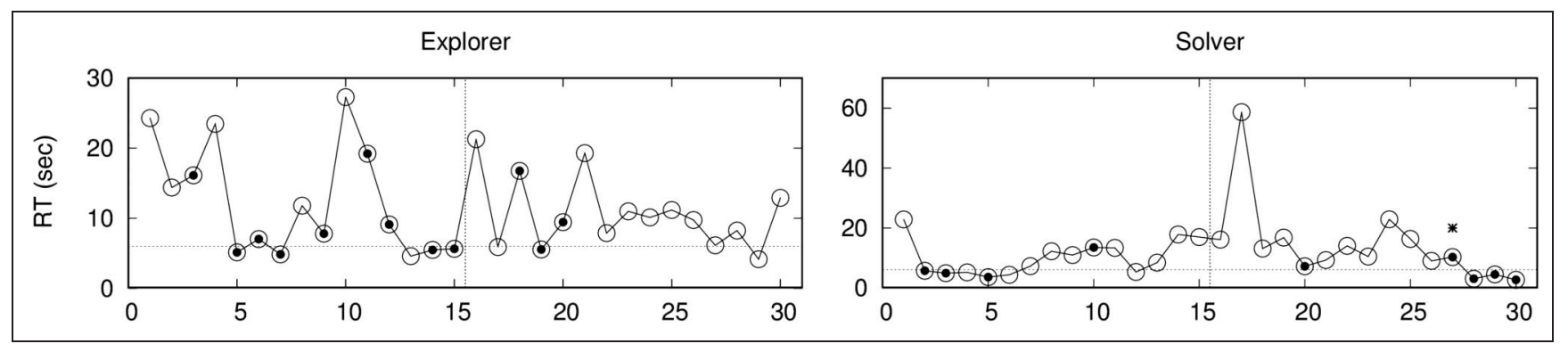

Figure 3. Behavioral data of an Explorer and a Solver. RT is averaged over moves within a trial and plotted for all trials. The winning trials are filled with black circles. The vertical dashed line separates the first and the second game. The horizontal dashed line is the 6-sec threshold for RT decline. The switch trial is indicated with an asterisk.

in mathematics in the national university entrance examination. Similar to the sex covariate, we introduced accuracy as a covariate of no interest using ANCOVA. Separately, we explored the effect of accuracy alone in a regression analysis. Similarly, we analyzed the effect of mathematical ability in two ways: first, as a covariate of no interest in an ANCOVA and, second, by balancing the mathematical scores in the two groups by removing nine participants with the top math scores (all were Solvers) so that the two groups have comparable scores. In all analyses, a threshold mask of 0.1 was used to remove the background noise and global normalization was omitted. The results were corrected for multiple comparisons at $p \leq .05$ using family-wise error correction.

\section{RESULTS}

\section{Behavioral Results}

Seventy-six healthy adults (41 women), 20-32 years old $(22.61 \pm 2.2$ years, female age $=22.90 \pm 2.41$ years, male age $=22.26 \pm 1.95$ years $)$, participated in the study. Fortyone participants (14 women) were categorized as Solvers and 35 participants ( 27 women) as Explorers. The behavioral plots of one Explorer and one Solver are shown in
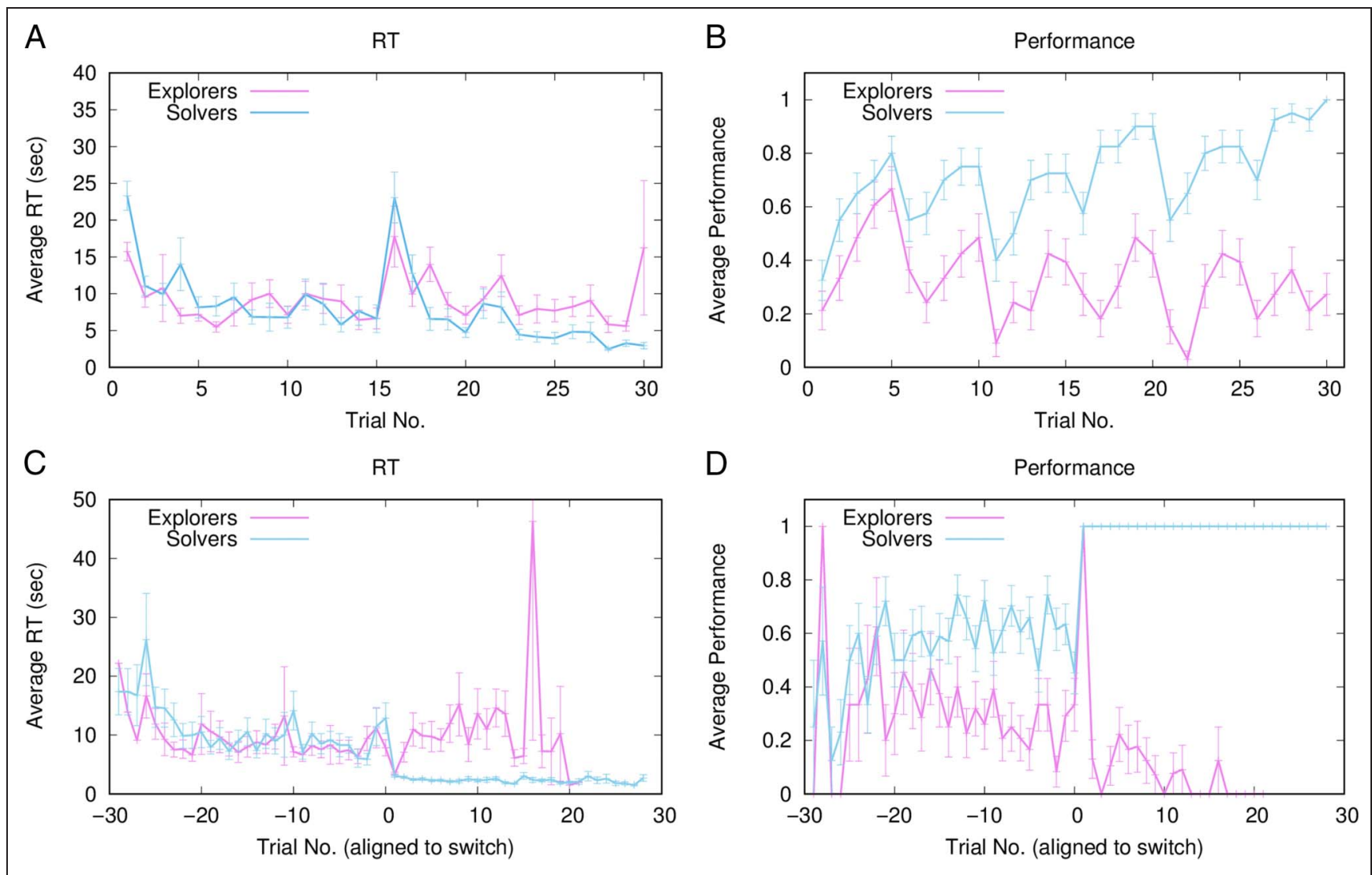

Figure 4. Average RTs and performance. (A, B) The time series of RT and performance averaged trial by trial in Explorers and Solvers. (C, D) The time series of performance and RT in Solvers and Explorers are aligned to their individual switch trial for Solvers and the hypothetical switch trial for Explorers. 


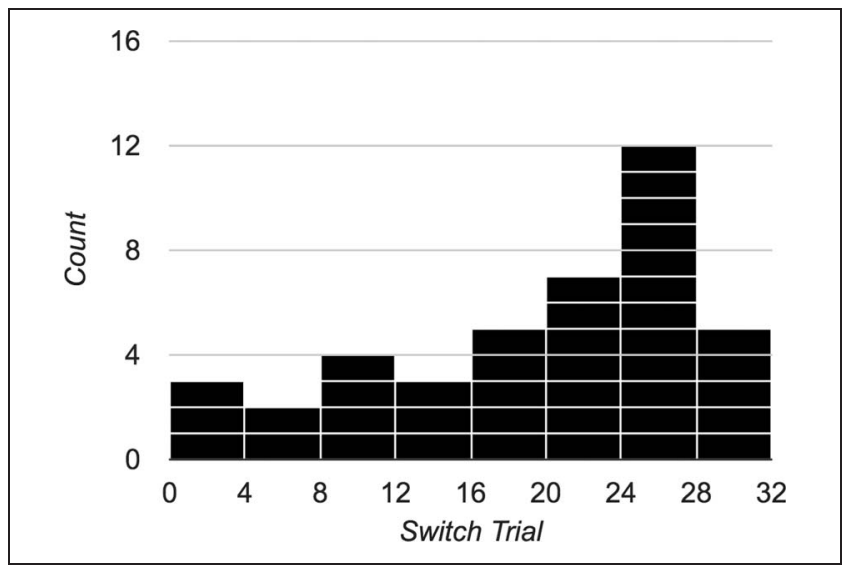

Figure 5. The histogram of switch trial in Solvers.

Figure 3. There was no significant age difference between Solvers and Explorers (two-tailed $t$ test, $t=0.25, p=.8$ ), but there was a significant sex difference in proportion of Solvers and Explorers (Fisher exact test, $p<.0001$ ).

To show the typical behavior of Solvers, we aligned the time series of all Solvers to the switch trial to compute average RT and performance. To compare Solvers and Explorers, we aligned the Explorers' RT and performance to a hypothetical switch point that was defined as follows: the start of the last sequence of wins in which RT is also below the 6-sec threshold. The first trial of the second game is removed from this analysis. In Figure 4, the time series of average RT and performance are plotted. Consistently successful performance and RT decline (by definition) as reflected in the time series after restructuring.

On average, Solvers took around $21.47(S D=7.13)$ trials to find the winning strategy. The distribution of switch trials is shown in Figure 5. Before a strategy shift occurs, Solvers played correctly on $60.4 \%$ of the trials $(S D=$ 16.96) and took in average $11.64 \mathrm{sec}$ per move $(S D=$ 10.08). Explorers played correctly in $32.63 \%$ of the trials $(S D=16.72 \%)$ and took in average $9.25 \mathrm{sec}$ per move $(S D=6.31)$. Thus, Solvers landed on critical positions at a higher rate. The difference in performance before the switch is significant ( $t$ test, $t=7.08, p<.0001$ ), but the difference in RT is not significant (11.45 vs. 9.25, $S D=10.08$ vs. $6.31, t$ test, $t=1.43 ; p=.15)$. Inspection of Figure 4 shows that over the first trials 1-4 Solvers performed little better than Explorers (53.57 vs. $40.91, S D=28.99$ vs. $26.38, t$ test, $t=1.95, p=.054)$. Moreover, the efficiency (accuracy/RT) of the two groups was equivalent ( $t$ test, $t=0.09, p=.92$ ). There is no significant difference between RT in trials $1-4$ ( $t$ test, $t=1.7$, $p=.9)$. However, the Solvers took longer than Explorers to make their moves in losing trials ( $t$ test, $t=2.81, p=$ .0055 ). In addition, male Solvers (mean switch trial 19.07, $S D=7.69)$ discovered the correct strategy earlier than female Solvers (mean switch trial 24.92, $S D=5.54, t$ test, $p<.004)$. The distribution of switch trials for Solvers is plotted in Figure 5.

To understand if Solvers could be considered better problem solvers in general, in a follow-up analysis we collected mathematics and physics grades of the national university entrance examination for 66 available participants (Figure 6). The top 9 mathematics scores belonged to Solvers. The difference between Solvers and Explorers is significant for Mathematics (two-tailed $t$ test, $t=2.60$, $p=.01$ ) but not Physics grades (two-tailed $t$ test, $t=$ 1.50, $p=.13)$.

\section{Voxel-based Morphometry Analysis}

An initial test was carried out to compare the gray matter volume of the Solvers versus Explorers (Figure 7). Voxels with family-wise error corrected $p$ values less than or equal to .05 are reported in Table 1 . Significant differences were found in the left angular/supramarginal gyrus, right precuneus, right angular gyrus, and the mid temporal gyrus in the left hemisphere. Most importantly, gray matter volume was significantly higher in Solvers in the right but not the left inferior frontal gyrus (BA 47). No significant differences were obtained for Explorers versus Solvers. The same comparison for the white matter volume shows significant difference in the left occipital and in the right parietal lobe (Figure 7; Table 1) for Solvers versus Explorers. The reverse contrast did not show any significant differences.

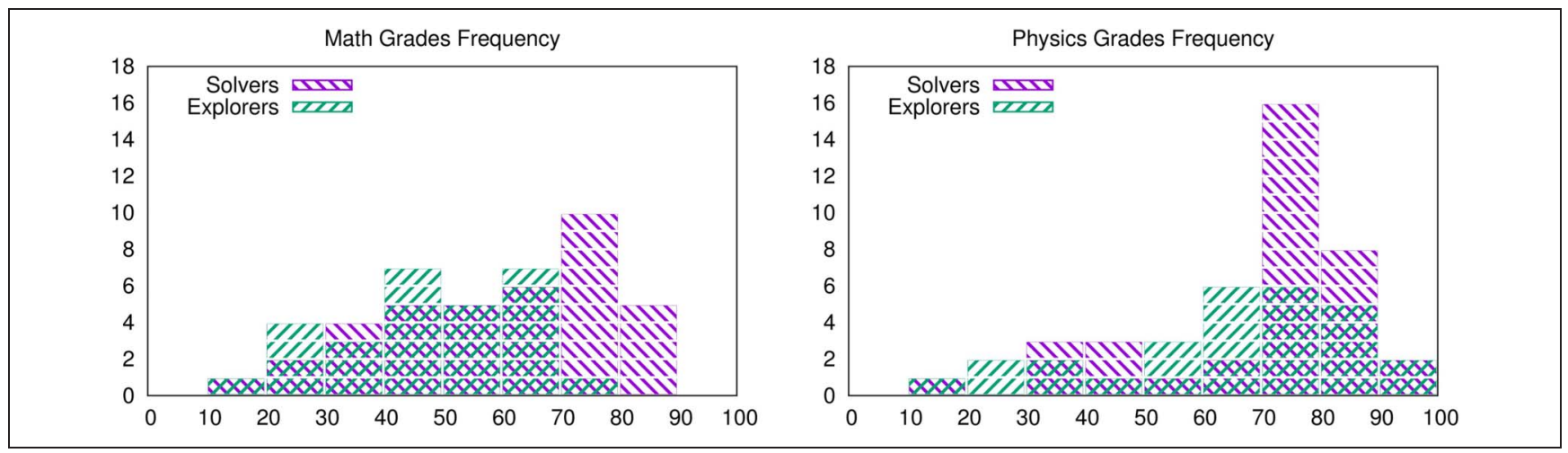

Figure 6. The histogram of Mathematics and Physics grades of Solvers and Explorers in the national university entrance examination. 
Figure 7. Brain areas where gray and white matter volumes were higher in Solvers versus Explorers. A threshold of $T>$ 3.8 was used for visualization purposes. See Table 1 for statistical values.

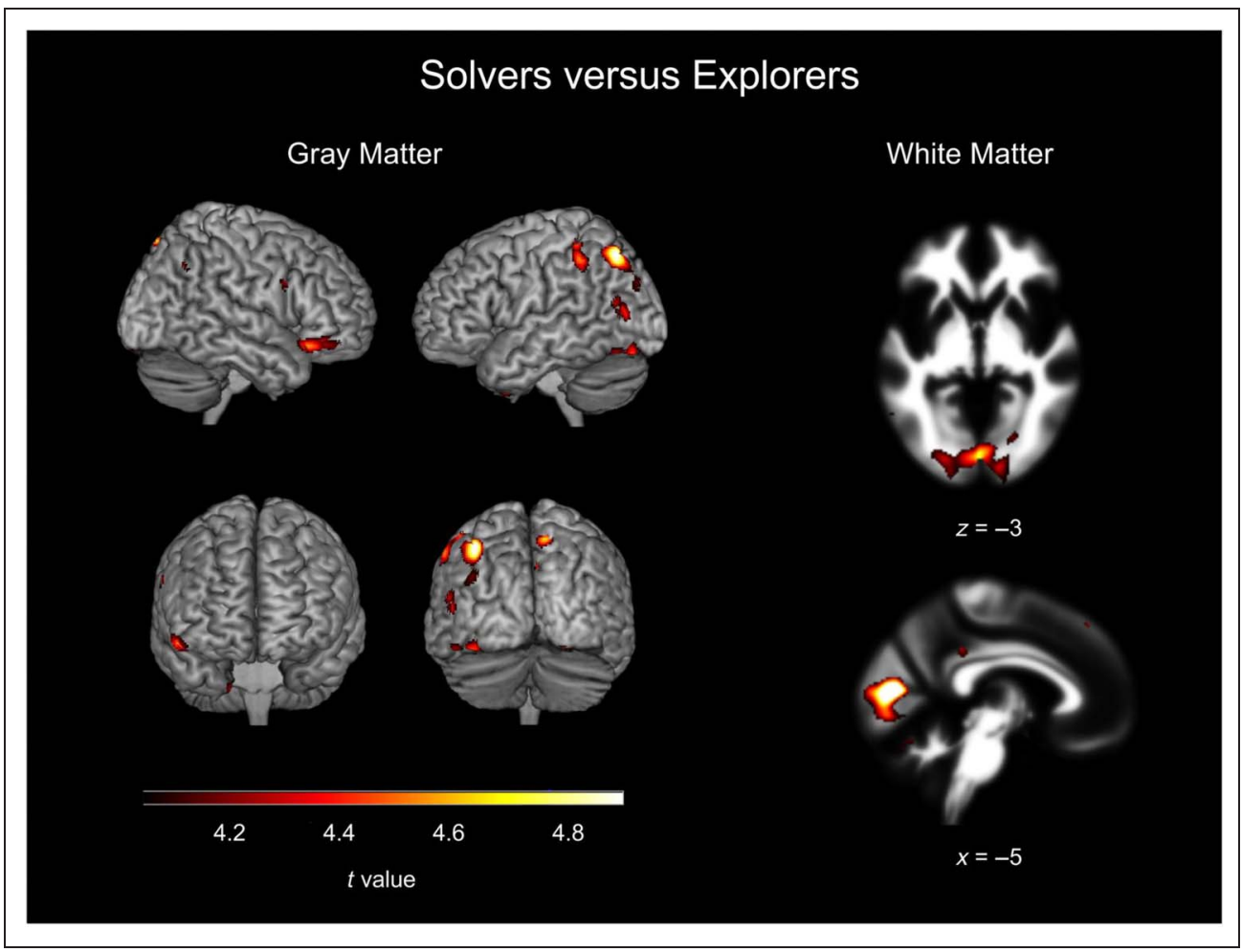

Given the sex difference observed in the behavior, the observed correlation might be related to differences of brain structure between the two sexes. To discover whether the differences found between Solvers and Explorers were entirely due to sex or not, we included sex as covariates of no interest in a second analysis using ANCOVA. The results are reported in Figure 8 and
Tables 2 and 3. Reassuringly, partialing out the impact of sex substantially reinforced the results. In Solvers, gray matter volume was higher in a swathe of areas in MPFC including BA 10, BA 11, and BA 32 extending to bilateral BA 47. Similarly, white matter volume in Solvers was higher around MPFC (BA 10 and BA 11), an area surrounded by BA 9, BA 8, and BA 32 and bilateral areas close to BA 47.

Table 1. MNI Coordinates for Voxels Showing Significantly Higher Gray/White Matter Volume in Solvers versus Explorers ( $p \leq .05$, FWE-corrected) Given in Millimeters with Effect Sizes (z Scores)

\begin{tabular}{|c|c|c|c|c|c|c|c|}
\hline Region & $B A$ & $x$ & $y$ & $z$ & z Score & Cluster Size & $p_{F W E-c o r r}$ \\
\hline \multicolumn{8}{|c|}{ Solvers vs. Explorers (Gray Matter Volume) } \\
\hline $\mathrm{L}$ angular gyrus & 7 & -38 & -69 & 51 & 4.52 & 157 & .01 \\
\hline $\mathrm{R}$ precuneus & 7 & 9 & -67 & 37 & 4.56 & 126 & .01 \\
\hline $\mathrm{R}$ precuneus & 23 & 14 & -58 & 27 & 4.42 & 126 & .02 \\
\hline $\mathrm{R}$ angular gyrus & $39 / 40$ & 40 & -51 & 33 & 4.44 & 24 & .02 \\
\hline L mid temporal gyrus & 39 & -58 & -70 & 16 & 4.27 & 3 & .04 \\
\hline $\mathrm{R}$ precuneus & 7 & 10 & -78 & 57 & 4.24 & 2 & .05 \\
\hline $\mathrm{R}$ inferior frontal gyrus & 47 & 54 & 28 & -17 & 4.24 & 1 & .05 \\
\hline \multicolumn{8}{|c|}{ Solvers vs. Explorers (White Matter Volume) } \\
\hline L occipital lobe & BA 18 & -3 & -79 & 9 & 5.41 & 443 & $<.001$ \\
\hline $\mathrm{R}$ parietal lobe & BA 7 & 16 & -69 & 51 & 4.40 & 2 & .04 \\
\hline
\end{tabular}

Nearest cortical area are reported for white matter. 
Figure 8. The difference of gray matter and white matter volume in Solvers versus Explorers including the sex covariates of no interest. A threshold of $T>5$ was used for visualization purposes. See Tables 2 and 3 for statistical values.

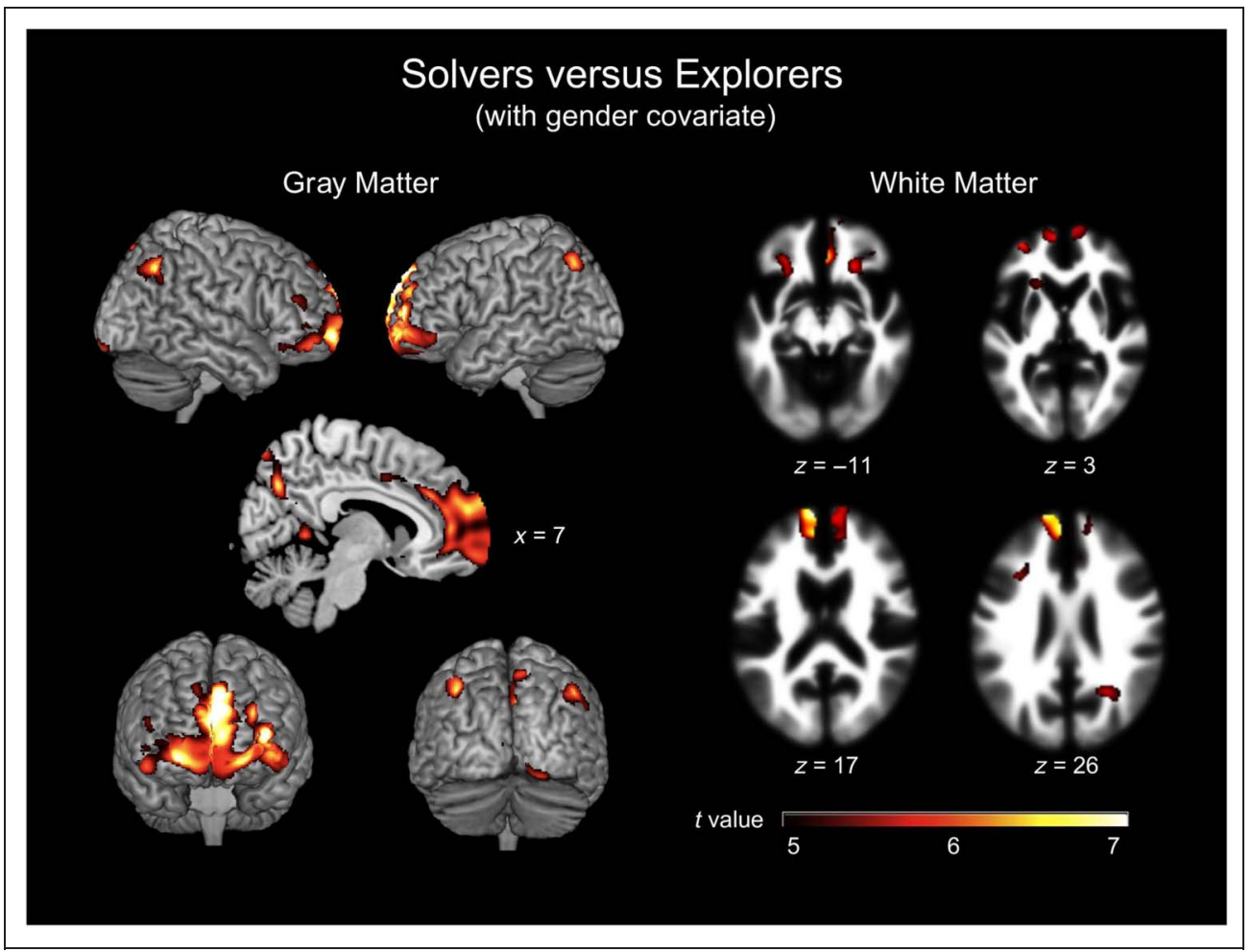

Direct comparison of male Solvers with female Solvers indicated several regions that were significantly different in gray matter volume. These regions included the left parietal and the right BA 47. In addition, mid temporal, bilateral BA 46, and dorsolateral pFC were different in male versus female Solvers. However, only the mid temporal region was also present when comparing male Explorers with female Explorers. No region was found for the reverse contrasts, that is, female versus male (Solvers/Explorers), and also no region was found for the interactions.

Because Solvers performed better than Explorers even before the switch, one possible explanation is that the number of correct trials alone and not solving per se could account for the difference we observed. To explore this possibility, we did a multiple regression analysis with accuracies on structural images. For Solvers, we used average performance before the switch, and for Explorers, the average performance in all trials. The overall result is weaker for accuracy regression, and only the left parietal cluster survives the multiple comparison correction $(x=$ $-38, y=-88, z=28 ; \mathrm{z}=4.23, k=4, p=.04)$. Although accuracy and solving are highly correlated, the small overlap between the groups (Explorers that performed better and Solvers that performed worse before switch) led to the loss of frontal findings. We have also investigated the effect of a measure of performance that is less correlated with Solving but might be causally related to Solving. A similar multiple regression analysis was done with the number of wins before restructuring for Solvers and the total number of wins for Explorers. No significant cluster was present, strengthening our original claim that preswitch performance measures on their own cannot account for the frontal Solving localizations. In another analysis, we compared Solvers and Explorers by introducing accuracy before switch as a covariate of no interest using ANCOVA. The gray matter volume in the left BA 47 (peak: $x=36, y=24, z=-17$; $z=4.89$, $p=.008)$ and all the posterior regions including the parietal regions (peak: $x=-36, y=-67, z=51 ; z=5.21$, $p=.002$ ) survived this analysis. Additionally, we found a difference in MPFC (peak: $x=-14, y=35, z=-12$; $\mathrm{z}=$ $4.55, p=.033$ ) and the left frontopolar cortex (peak: $x=$ $-38, y=63, z=12 ; \mathrm{z}=5.58, p<.001)$.

To find out whether general problem-solving ability could explain the difference between Solvers and Explorers, we added mathematics scores as a covariate of no interest using ANCOVA. The results were similar to the sex analysis and the network of regions found previously survived in this analysis as well. Additionally, we found that the top 9 mathematics scores belong to Solvers. Removing these participants resulted in Solvers and Explorers with similar average math scores (49.5 \pm 17.59 vs. $46.41 \pm 16.30 ; t$ test, $t=0.69, p=.49$ ). By comparing the Solvers and Explorers in this analysis, again the left parietal (peak: $x=-38, y=-70, z=56$; $\mathrm{z}=4.46$, $p=.025$ ) and right BA 47 (peak: $x=45, y=38, z=-5$; $\mathrm{z}=4.44, p=.028$ ) survived the statistical threshold. A summary of these analysis and their results are shown in Table 4. 
Table 2. MNI Coordinates for Significant Gray Matter Voxels in Solvers versus Explorers Including Sex as Covariate of No Interest Using ANCOVA ( $p \leq .05$, FWE-corrected) Given in Millimeters with Effect Sizes (z Scores)

\begin{tabular}{|c|c|c|c|c|c|c|c|}
\hline \multicolumn{8}{|c|}{$\begin{array}{c}\text { Solvers vs. Explorers Using Sex Covariate } \\
\text { (Gray Matter Volume) }\end{array}$} \\
\hline \multirow[b]{2}{*}{ Region } & \multirow[b]{2}{*}{$B A$} & \multirow[b]{2}{*}{$x$} & \multirow[b]{2}{*}{$y$} & \multicolumn{3}{|c|}{ Cluster } & \multirow{2}{*}{$\begin{array}{l}p_{F W E-} \\
\text { corr }\end{array}$} \\
\hline & & & & $z$ & Size & $z$-Score & \\
\hline $\begin{array}{l}\text { L superior medial } \\
\text { gyrus }\end{array}$ & 10 & -6 & 66 & 28 & 31584 & 6.70 & $<.001$ \\
\hline $\begin{array}{l}\text { L superior medial } \\
\text { gyrus }\end{array}$ & 9 & -5 & 54 & 38 & 31584 & 6.30 & $<.001$ \\
\hline $\begin{array}{l}\text { L superior medial } \\
\text { gyrus }\end{array}$ & 32 & -14 & 35 & 27 & 31584 & 6.00 & $<.001$ \\
\hline L ACC & 24 & -2 & 26 & 35 & 31584 & 5.87 & $<.001$ \\
\hline L ACC & 32 & 0 & 35 & 30 & 31584 & 5.83 & $<.001$ \\
\hline $\begin{array}{l}\text { L superior orbital } \\
\text { gyrus }\end{array}$ & 11 & -17 & 51 & -8 & 31584 & 5.73 & $<.001$ \\
\hline $\begin{array}{l}\text { R superior medial } \\
\text { gyrus }\end{array}$ & 32 & 9 & 54 & 24 & 31584 & 5.69 & $<.001$ \\
\hline $\begin{array}{l}\text { L superior frontal } \\
\text { gyrus }\end{array}$ & 46 & -29 & 56 & 26 & 31584 & 5.68 & $<.001$ \\
\hline $\mathrm{R}$ angular gyrus & 39 & 45 & -60 & 42 & 1426 & 6.03 & $<.001$ \\
\hline $\mathrm{R}$ precuneus & 7 & 8 & -68 & 38 & 1653 & 5.80 & $<.001$ \\
\hline $\mathrm{R}$ precuneus & 23 & 12 & -56 & 27 & 1653 & 4.98 & .005 \\
\hline $\mathrm{L}$ angular gyrus & 7 & -38 & -68 & 45 & 825 & 5.75 & $<.001$ \\
\hline $\begin{array}{l}\text { R middle frontal } \\
\text { gyrus }\end{array}$ & 45 & 45 & 41 & 20 & 273 & 4.95 & .006 \\
\hline $\begin{array}{l}\text { L superior } \\
\text { temporal gyrus }\end{array}$ & 48 & -44 & -9 & -9 & 41 & 4.77 & .013 \\
\hline $\begin{array}{l}\text { L middle frontal } \\
\text { gyrus }\end{array}$ & 45 & -32 & 41 & 17 & 11 & 4.67 & .02 \\
\hline L calcarine gyrus & 17 & -5 & -101 & 8 & 39 & 4.65 & .02 \\
\hline $\begin{array}{l}\text { L cerebelum } \\
\quad(\text { Crus } 1)\end{array}$ & 19 & -50 & -68 & -17 & 41 & 4.9496 & .02 \\
\hline $\begin{array}{l}\text { L inferior parietal } \\
\quad \text { lobule }\end{array}$ & 40 & -60 & -44 & 44 & 4 & 4.46 & .04 \\
\hline
\end{tabular}

\section{DISCUSSION}

In this article, we employed a novel behavioral approach with the aim of producing evidence on the anatomical correlates of what we have characterized as the major problem restructuring process. Our paradigm fulfilled four properties (Seyed-Allaei et al., 2010):

I. Problem restructuring was preceded by an initial impasse in problem solving. Choices were consistently slow and unsuccessful during this period.
Table 3. MNI Coordinates for Significant White Matter Voxels in Solvers versus Explorers Including Sex as Covariate of No Interest Using ANCOVA ( $p \leq .05$, FWE-corrected) Given in Millimeters with Effect Sizes (z Scores)

\begin{tabular}{lrrrrrr}
\hline \multicolumn{7}{c}{$\begin{array}{c}\text { Solvers vs. Explorers } \\
\text { (White Matter Volume) }\end{array}$} \\
\hline Nearest BA & $x$ & $y$ & $z$ & Cluster Size & z-Score & $p_{F W E-c o r r}$ \\
\hline 10 & -12 & 62 & 21 & 2427 & 6.22 & $<.001$ \\
11 & 3 & 35 & -14 & 3750 & 5.71 & $<.001$ \\
47 & -29 & 23 & -5 & 2463 & 5.70 & $<.001$ \\
$18 / 19$ & 26 & -57 & 25 & 646 & 5.11 & .002 \\
32 & 9 & -20 & 45 & 87 & 5.10 & .002 \\
17 & -10 & -81 & 7 & 240 & 4.84 & .006 \\
48 & 38 & -16 & 13 & 53 & 4.72 & .011 \\
47 & 35 & 42 & 6 & 84 & 4.64 & .015 \\
19 & 26 & -67 & -3 & 29 & 4.53 & .023 \\
11 & -6 & 41 & 3 & 15 & 4.49 & .027 \\
7 & 16 & -67 & 51 & 7 & 4.49 & .027 \\
$24 / 32$ & -8 & 23 & 30 & 13 & 4.47 & .029 \\
$19 / 21 / 37$ & 36 & -49 & 9 & 37 & 4.46 & .031 \\
\hline & & & & & & \\
\hline
\end{tabular}

II. Solving the problem entailed a significant novel understanding of the problem description (Figure 2) that corresponded to major restructuring of the problem state space-which corresponds to Goel's (1995) "lateral transformations."

III. The restructuring was achieved suddenly. Choices were consistently fast and successful after restructuring.

IV. Substantial individual differences were observed between participants; nearly half of our participants did not succeed in figuring out the solution in 30 trials.

Table 4. Summary of Regions with Significantly More Gray Matter Volume in Different Analyses

\begin{tabular}{|c|c|c|c|c|}
\hline $\begin{array}{l}\text { Solvers vs. } \\
\text { Explorers }\end{array}$ & $\begin{array}{c}\text { R Inferior } \\
\text { Frontal } \\
\text { Gyrus }\end{array}$ & $\begin{array}{l}\text { Medial } \\
\text { Frontal }\end{array}$ & $\begin{array}{c}\text { L Angular } \\
\text { Gyrus/ } \\
\text { Intraparietal }\end{array}$ & $R$ Precuneus \\
\hline $\begin{array}{l}\text { Without } \\
\text { covariates }\end{array}$ & $\mathrm{X}$ & - & $\mathrm{X}$ & $\mathrm{X}$ \\
\hline $\begin{array}{l}\text { Accuracy } \\
\text { ANCOVA }\end{array}$ & $\mathrm{X}$ & $\mathrm{X}$ & $\mathrm{X}$ & $\mathrm{X}$ \\
\hline Math ANCOVA & $\mathrm{X}$ & $\mathrm{X}$ & $\mathrm{X}$ & $\mathrm{X}$ \\
\hline $\begin{array}{l}\text { Excluded high } \\
\text { Math scores }\end{array}$ & $\mathrm{X}$ & - & $\mathrm{X}$ & - \\
\hline $\begin{array}{l}\text { Sex } \\
\text { ANCOVA }\end{array}$ & $\mathrm{X}$ & $\mathrm{X}$ & $\mathrm{X}$ & $\mathrm{X}$ \\
\hline
\end{tabular}


These four features ensured that our paradigm was well suited for assessing the localization of systems underlying major problem restructuring using neuroimaging based on individual differences (Kanai \& Rees, 2011). However, the cognitive processes responsible for many aspects of carrying out the specific task other than those involved specifically in major problem restructuring will also be picked up by our methodology. In particular, carrying out the task involves some very basic spatial processing and, more critically, simple arithmetic. Each player can move between 1 and 3 squares at each turn and the critical rule that has to be discovered involves realizing that critical squares are four (i.e., $1+$ maximum step size) apart. Moreover, major problem restructuring may depend in part on processes occurring before the event itself, which we will call pre-restructuring ones (see below).

To differentiate the neuronal substrates linked to general aspects of task execution versus major problem restructuring versus pre-restructuring, we carried out a number of subsidiary analyses. We covaried for the effect overall accuracy of the participant (before major restructuring). We also controlled for mathematical ability in two different ways. We first added mathematical ability as a covariate to the general linear model. In a further analysis, as the nine highest participants on mathematical ability were all Solvers, we removed these participants, which left the Modified Solver group at close to the mathematical ability of the Explorer group, and then compared the Modified Solver and Explorer groups directly.

Our results showed that three regions at least may be critically involved in successfully carrying out the taskleft inferior parietal area, the right precuneus, and the right inferior frontal area. The left inferior parietal area appears to stretch from the inferior parietal lobule into the superior parietal lobule. This is, of course, a primary area for number processing and also for calculation (Arsalidou \& Taylor, 2011; Hubbard, Piazza, Pinel, \& Dehaene, 2005; Dehaene, Spelke, Pinel, Stanescu, \& Tsivkin, 1999). However, the involvement of this area in our study survives covarying for overall accuracy and mathematical ability and also is found when the Modified Solver group is used. Thus, it may well be involved in the major problem restructuring process itself or pre-restructuring. A second area that survived the statistical threshold of significance was the right precuneus. However, when we excluded the high math score subjects to produce the Modified Solver group, the right precuneus region is no longer found. We will therefore not consider it further.

The one frontal area where solving the problem was significantly correlated with gray matter volume was indeed right ventrolateral pFC (BA 47). In the Arsalidou and Taylor (2011), meta-analysis only considerably higher regions of the right lateral frontal cortex were involved in either number processing or calculation. Thus, even a task like the Brixton Spatial Anticipation test where good performance requires both spatial and abstraction processes as well as numerical ones does not give rise to BA 47 activations (Crescentini et al., 2011).
By contrast, the BA 47 area found in our study was close to the coordinates, also in BA 47, obtained by Goel and Vartanian (2005) and Vartanian and Goel (2005) in their functional activation studies of Matchstick Problems and anagram problems, respectively. This was where they held that systems responsible for lateral transformations were located. Thus, our results corroborated the prediction of Goel and Vartanian and indirectly provide some support for their interpretation of their findings.

However, two aspects of the behavioral results produced complications for the simple picture that systems in the right lateral prefrontal (BA 47) are where the critical processes responsible for major problem restructuring are located. First, as alluded to earlier, there are differences in the performance of the Solvers and Explorers groups before any strategy switch. Second, there is a prominent sex effect.

Thus, major problem restructuring appears to show up as an all-or-none change in behavior at a certain timethat of the "Aha" experience. The first complication for the simple interpretation of the role of BA 47 presented above is that any process occurring at this time is likely to have been preceded by other related ones. It is well known that, in difficult problem solving, conscious and possibly unconscious mental processes like incubation may facilitate the occurrence of a later insightful leap (Sio \& Ormerod, 2009; Woodworth \& Schlosberg, 1955). Thus, Duncker (1935) alludes to the solution of complex problems being preceded by steps with increasing degrees of "partial insight." Ruger (1910) in his study of insightful problem solving describes so-called "locus analysis" where the participant notices the place or part of a puzzle where success has occurred on some trials. Our observations are consistent with these ideas. Many participants learn to land on the critical squares before they solve the problem exhaustively. Moreover, in our study the longer a participant takes to consider her options before a strategy switch, the better is the opportunity to learn which the critical squares are.

Indeed, in the current study the performance of Solvers and Explorers differs before the strategy switch point as well as after it. The accuracy of Solvers is better than that of Explorers even before the restructuring point. It is therefore highly plausible that critical prerestructuring processes differ between the two groups. More specifically, the two groups show comparable success rates over the first few trials. However, Solvers take longer over these unsuccessful initial trials. After that performance diverges. This finding offers an interesting possibility about the difference in the cognitive basis of problem solving in Solvers and Explorers. When in the initial trials, Solvers take more time they perhaps think more deeply about the problem. Explorers, on the other hand, seem to approach the task by trial and error, trying out one action after another at a steady rate. In any case, Solvers land on the critical squares at a higher rate than do Explorers. They may be said to be more effective at 
Ruger's "locus analysis." It is therefore highly plausible that critical pre-restructuring processes differ between the two groups.

With the current methodology, one cannot differentiate the neural correlates of major problem restructuring processes from any pre-restructuring ones. However, one can examine whether measures that are more closely linked to hypothetical pre-restructuring processes than restructuring ones themselves give similar functional localizations to those found in the basic analyses. When this is done using the average performance before a switch as the measure or using the number of correct trials, then left parietal effects are found for average performance before the switch but no frontal effects are found. This suggests that the left parietal region may contribute to the pre-restructuring processes. Indirectly, it makes the linking of major problem restructuring processes themselves to the BA 47 region more plausible. It, however, does not rule out the possibility that the variability in the left parietal region signifies cognitive processes concerned with major problem restructuring itself. Indeed, the involvement of a network of brain areas in such processes is highly plausible. This is consistent with what is known about parietal cortex's role in numerical cognition. In a task where the novel strategy involves calculation, regions critical for calculation must be involved for problem restructuring, unless the processing they undertake is completely mimicked in more frontal regions, which seems unlikely.

There was a second complication that needs to be taken into account before accepting the simple picture that systems in the right ventrolateral $\mathrm{pFC}$ (BA 47) are where the critical processes responsible for major problem restructuring are located. Male participants comprised 66\% $(27 / 41)$ of the Solvers, but only $23 \%$ (8/35) of the Explorers. In addition, male Solvers arrived at the solution with less trials on average than do female Solvers. This raises the possibility that sex constitutes a confound for our measure of problem restructuring and the results reported above could be influenced by the difference in sex distribution. In a further analysis, we used ANCOVA to partial out the impact of sex by including sex as a covariate in our general linear model. If our results were primarily due to the difference in sex distributions, then the correlation between gray matter volume in right BA 47 and behavioral label (Solver vs. Explorer) should be wiped out or substantially reduced. In fact, the results were substantially strengthened (Tables 2 and 3; Figure 8). Once the confounding effects of sex were removed, a long vertical swathe of anterior medial rostral pFC (BA 10 and BA 11) and two roughly symmetrical extensions bilaterally into BA 47 showed strong correlations with problem restructuring. Most importantly, in addition to the two posterior regions found in the basic analysis, the peak BA 47 voxel found in the first analysis was within this extension into BA 47 as was the equivalent region in left BA 47. This analysis therefore provided evidence that our findings were not confounded by sex distribution.

Two additional analyses supported this idea. The comparison of male Solvers with female Solvers showed that gray matter differences were found in a number of regions including both the right ventrolateral frontal and the left inferior parietal. This is compatible with male Solvers finding a solution on average before female Solvers. However, when male Explorers were compared with female Explorers, neither of these regions were among those that show differences. However, it should be noted that no regions were found when the interaction was tested.

We have argued that the left inferior parietal region would be expected to be involved in problem restructuring with this task. Why should bilateral BA 47 and the anterior medial rostral pFC swathe also be regions involved in succeeding at the Subtraction Game? It is important to distinguish between the two areas in several respects. First, the anterior medial rostral pFC swathe is a central part of the default network; by contrast, BA 47 is not part of its core systems (Buckner, Andrews-Hanna, \& Schacter, 2008). Second, a recent work (Schuck et al., 2015) also reported that the information related to new strategy in a perceptual task is encoded in the human medial pFC (but not BA 47) several blocks before and not after the change. Schuck et al. argued that the dorsolateral pFC favors task relevant information (e.g., Duncan, 2001; Miller $\&$ Cohen, 2001). They suggest that the anterior medial rostral pFC escapes this bias and so codes task-irrelevant information. This latter proposition could also be relevant to our study. To change strategy in our task, participants must "zoom out" from the specific details of the game and note the critical importance of reaching the fixed landmark positions (e.g., positions 3, 7, and 11 in Figure 2) for winning. The cognitive process underlying such "zooming out" may involve medial rostral pFC. For example, Burgess, Dumontheil, and Gilbert (2007) have proposed with much evidence that the anterior medial rostral $\mathrm{pFC}$ acts as a gateway system to control whether attention to task is externally or internally focused. One possibility is that systems in the anterior medial rostral $\mathrm{pFC}$ facilitate escape from the specific details of the game, that is, the currently dominant thought processes to infer the higher-level winning strategy. This proposition also fits with medial rostral pFC involvement in mind-wandering or stimulusindependent thought (Buckner et al., 2008; Mckiernan, Kaufman, Kucera-Thompson, \& Binder, 2003; McGuire et al., 1995).

What are the processes involved in major problem restructuring? Assume, using the terminology of Norman and Shallice (1986), that a strategy is implemented by one or a set of thought or actions schemas-like productions (Newell \& Simon, 1972) but with an added valence variable, which is critical for selection when triggered schema conflict. So an Explorer strategy would correspond to the four schemas shown in Table 5 (a). By 
Table 5. Strategy Schemas of Explores and Solvers

\begin{tabular}{|c|c|c|}
\hline & (a) Explorer Strategy Schemas & (b) Solver Strategy Schemas \\
\hline 1 & $\begin{array}{l}\text { If player is to play and position pawn }=\mathrm{p} \text {; activate } \\
\text { schema (ascertain estimated valence for position } \\
(\mathrm{p}+i) \text { where } i=1,2,3)\end{array}$ & $\begin{array}{l}\text { If player moves to position pawn to position p: activate } \\
\text { schema (Is valence at p very high?) }\end{array}$ \\
\hline 2 & $\begin{array}{l}\text { Then: activate schema (select highest estimated } \\
\text { valences }(p+1, p+2, p+3))\end{array}$ & If Yes: activate schema (store position $\mathrm{p}$ in a buffer?) \\
\hline 3 & $\begin{array}{l}\text { If current round finished and if player wins: activate } \\
\text { schema (increase valence of all intermediate } \\
\text { positions visited) }\end{array}$ & $\begin{array}{l}\text { At some point: activate schema (Is there an arithmetic } \\
\text { relation between positions stored in the High Valence } \\
\text { buffer?) }\end{array}$ \\
\hline 4 & $\begin{array}{l}\text { If current round finished and if player loses: activate } \\
\text { schema (decrease valence of all intermediate } \\
\text { positions visited) }\end{array}$ & $\begin{array}{l}\text { If yes: activate schema (Use relation to determine } \\
\text { response) }\end{array}$ \\
\hline
\end{tabular}

contrast, a Solver strategy would correspond to the four schemas shown in Table 5 (b).

There are thus at least four thought schemas present in the Solver strategy procedure but not the Explorer strategy one. Which is the critical one for the BA 47 activation remains to be established. However, if one considers the first two Solver schemas, although they are not necessary for the Explorer strategy, they could easily provide a useful addition to it and are naturally related to its schemas. Solver schema 2 involves the additional use of a buffer to hold high valence positions; this could well involve the inferior parietal regions, strong candidates for the location of the phonological input buffer on the Baddeley-Hitch working memory model (Shallice \& Cooper, 2011; Baldo \& Dronkers, 2006; Paulesu et al., 1993). By contrast, Solver schema 3 is unrelated to the Explorer schemas. As important, it is qualitatively different. Most critically, it lacks the production system characteristic of having its if-specified component strongly linked to the recently added contents of working memory. What triggers its activation at any particular time is not transparent. It is this temporal nontransparency, which makes it the obvious information-processing correspondent of the phenomenological "insight." In the Matchstick Problem case, the temporally nontransparent process is the dropping from the schema (Count Squares) of an argument implicitly added such as (same size).

Which cognitive process contributes to major problem restructuring with its temporally nontransparent characteristics? Here, Goel et al.'s hypothesis about BA 47 is a strong candidate, which involved the formation of associations between previously unconnected sets of representations-high valence positions and arithmetic relations in the current case. This corresponds to the process that Amati and Shallice (2007) call latching and, in particular, the form coalescence. Convergence to the successful strategy typically follows after the participant has discovered the fixed landmark positions sufficient for winningSolver schemas 1 and 2 (see also Figure 2). In the coalescence process, an active temporary goal (say to determine what is the relation between the critical squares) combines with a supervisory process (say noticing they are four apart) to generate a new procedure (say count back in fours from the end square). We conjecture that the area BA 47 is specifically involved in this coalescence process. However, the anatomical nature of our results precludes us from being able to test this functional hypothesis here. Future research could combine this hypothesis with new approaches of fMRI analysis to further examine the role of area BA 47.

What can one make of the sex differences on the task and, in particular, the changes in lateralization in BA 47 according to how sex is treated in the analysis? An analysis of functional connectivity during resting state may provide insight on the latter point. Tomasi and Volkow (2012) found with their version of connectivity analysis in a study of 900 participants that the inferior frontal region is one of three cortical regions to show sex differences in lateralization. For this region, men showed higher rightward lateralization for short-range connectivity and women higher leftward lateralization for long-range connectivity. In our first analysis, men would dominate numerically the Solver activations, but this male bias is removed by the second analysis. This pattern maps well onto Tomasi and Volkow's findings, given that the cause of the better male performance on the task is linked to differences between sexes in their inferior frontal architecture, which remains to be conclusively demonstrated. The right dominance for the critical process would be more pronounced in men.

Finally, it is worth asking whether there is any possibility other than major problem restructuring for the involvement of BA 47 in our study. One may argue that figuring out the winning strategy is a form of abstraction. Chatham and Badre (2012) and Shallice and Cooper (2013) have argued that abstract semantic representations are stored (rather than computed as we have argued above) in the ventrolateral pFC. Importantly, 
neither study distinguished a lateralized role for the right frontal lobe, and indeed, Shallice and Cooper (2013) were concerned with word meanings that pertained to the left pFC. We believe that the currently available evidence (including the anatomical data presented here) is unable to distinguish between these possibilities.

\section{Conclusions}

There appear to be at least two differently localized processes involved in major problem restructuring. One of these, following Schuck et al., is located in the anterior medial rostral pFC; it is concerned with facilitating escape from the currently dominant thought processes. A second concerns the active restructuring process itself. The study provides strong support for the hypothesis put forward by Goel and colleagues on a key role for BA 47 in carrying out lateral transformations and somewhat weaker support for their lateralization claim, namely that it is the right BA 47 that is more critical for the restructuring process.

\section{Acknowledgments}

We would like to express our respects to our young collaborator, Hamideh Rastegar, who passed away recently in an accident. We thank Daniele Amati, Abdolhosein Abbasian, and Hamed Seyed-Allaei for their stimulating discussions and support. We thank Sohrab Shahzadi for helping us in creating the MRI database. This project was funded by the Institute for Research in Fundamental Sciences (IPM), Tehran, Iran. Bahador Bahrami was supported by the European Research Council (NEUROCODEC, StG Grant Number 309865). Shima SeyedAllaei was supported by Cognitive Sciences and Technologies Council of Iran.

Reprint requests should be sent to Shima Seyed-Allaei, Cognitive Systems Laboratory, School of Electrical and Computer Engineering, College of Engineering, University of Tehran, Tehran, Iran, or via e-mail: shima.allaei@gmail.com.

\section{REFERENCES}

Amati, D., \& Shallice, T. (2007). On the emergence of modern humans. Cognition, 103, 358-385.

Arsalidou, M., \& Taylor, M. J. (2011). Is $2+2=4$ ? Meta-analyses of brain areas needed for numbers and calculations. Neuroimage, 54, 2382-2393.

Ashburner, J. (2007). A fast diffeomorphic image registration algorithm. Neuroimage, 38, 95-113.

Baldo, J. V., \& Dronkers, N. F. (2006). The role of inferior parietal and inferior frontal cortex in working memory. Neuropsychology, 20, 529.

Bouton, C. L. (1901). Nim, a game with a complete mathematical theory. The Annals of Mathematics, 3, 35-39.

Buckner, R. L., Andrews-Hanna, J. R., \& Schacter, D. L. (2008). The brain's default network. Annals of the New York Academy of Sciences, 1124, 1-38.

Burgess, P. W., Dumontheil, I., \& Gilbert, S. J. (2007). The gateway hypothesis of rostral prefrontal cortex (area 10) function. Trends in Cognitive Sciences, 11, 290-298.

Chatham, C. H., \& Badre, D. (2012). Parts to principles: Anatomical origins of prefrontal organization. Cortex, 48, 1373-1375.
Crescentini, C., Seyed-Allaei, S., De Pisapia, N., Jovicich, J., Amati, D., \& Shallice, T. (2011). Mechanisms of rule acquisition and rule following in inductive reasoning. Journal of Neuroscience, 31, 7763-7774.

Dehaene, S., Spelke, E., Pinel, P., Stanescu, R., \& Tsivkin, S. (1999). Sources of mathematical thinking: Behavioral and brain-imaging evidence. Science, 284, 970-974.

Derrfuss, J., Brass, M., Neumann, J., \& von Cramon, D. Y. (2005). Involvement of the inferior frontal junction in cognitive control: Meta-analyses of switching and Stroop studies. Human Brain Mapping, 25, 22-34.

Duncan, J. (2001). An adaptive coding model of neural function in prefrontal cortex. Nature Reviews Neuroscience, 2, 820-829.

Duncker, K. (1935). Zur Psychologie des produktiven Denkens [Psychology of productive thinking]. Berlin: Springer.

Goel, V. (1995). Sketches of thought. Cambridge, MA: MIT Press.

Goel, V. (2009). Cognitive neuroscience of thinking. In G. G. Berntson \& J. T. Cacioppo (Eds.), Handbook of neuroscience for the behavioral sciences (pp. 417-430). New York: John Wiley \& Sons.

Goel, V., \& Grafman, J. (2000). Role of the right prefrontal cortex in ill-structured planning. Cognitive Neuropsychology, 17, 415-436.

Goel, V., \& Vartanian, O. (2005). Dissociating the roles of right ventral lateral and dorsal lateral prefrontal cortex in generation and maintenance of hypotheses in set-shift problems. Cerebral Cortex, 15, 1170-1177.

Guilford, J. P. (1967). The nature of human intelligence. New York, NY: McGraw-Hill.

Hawes, D. R., Vostroknutov, A., \& Rustichini, A. (2012). Experience and abstract reasoning in learning backward induction. Frontiers in Neuroscience, 6, 23.

Hubbard, M. E., Piazza, M., Pinel, P., \& Dehaene, S. (2005). Interactions between number and space in parietal cortex. Nature Review Neuroscience, 6, 435-448.

Kanai, R., Bahrami, B., \& Rees, G. (2010). Human parietal cortex structure predicts individual differences in perceptual rivalry. Current Biology, 20, 1626-1630.

Kanai, R., \& Rees, G. (2011). The structural basis of interindividual differences in human behaviour and cognition. Nature Reviews Neuroscience, 12, 231-242.

Knoblich, G., Ohlsson, S., \& Raney, G. E. (2001). An eye movement study of insight problem solving. Memory and Cognition, 29, 1000-1009.

Kounios, J., \& Beeman, M. (2014). The cognitive neuroscience of insight. Annual Review of Psychology, 65, 71-93.

Lee, N. Y. L., \& Johnson-Laird, P. N. (2013). Strategic changes in problem-solving. Journal of Cognitive Psychology, 25, 166-173.

Maguire, E. A., Gadian, D. G., Johnsrude, I. S., Good, C. D., Ashburner, J., Frackowiak, R. S. J., et al. (2000). Navigationrelated structural change in the hippocampi of taxi drivers. Proceedings of the National Academy of Sciences, U.S.A., 97, 4398-4403.

McGuire, P. K., Silbersweig, D. A., Murray, R. M., David, A. S., Frackowiak, R. S. J., \& Frith, C. D. (1995). Functional anatomy of inner speech and auditory verbal imagery. Schizophrenia Research, 15, 91-92.

Mckiernan, K. A., Kaufman, J. N., Kucera-Thompson, J., \& Binder, J. R. (2003). A parametric manipulation of factors affecting task-induced deactivation in functional neuroimaging. Journal of Cognitive Neuroscience, 15, 394-408.

Mechelli, A., Crinion, J. T., Long, S., Friston, K. J., Lambon Ralph, M. A., Patterson, K., et al. (2005). Dissociating reading processes on the basis of neuronal interactions. Journal of Cognitive Neuroscience, 17, 1753-1765.

Miller, E. K., \& Cohen, J. D. (2001). An integrative theory of prefrontal cortex function. Annual Review of Neuroscience, 24, 167-202. 
Miller, L. A., \& Tippett, L. J. (1996). Effects of focal brain lesions on visual problem-solving. Neuropsychologia, 34, 387-398.

Newell, A. \& Simon, H. A. (1972). Human problem solving. New Jersey, NJ: Prentice-Hall.

Norman, D. A., \& Shallice, T. (1986). Attention to action: Willed and automatic control of behavior. In R. Davidson, G. Schwartz, \& D. Shapiro (Eds.), Consciousness and self regulation: Advances in research and theory (pp. 1-18). New York, NY: Plenum.

Ohlsson, S. (1992). Information-processing explanations of insight and related phenomena. In M. Keane \& K. Gilhooley (Eds.), Advances in the psychology of thinking (pp. 1-44). New York: Harvester-Wheatsheaf.

Paulesu, E., Frith, C. D., \& Frackowiak, R. S. (1993). The neural correlates of the verbal component of working memory. Nature, 362, 342-345.

Ruger, H. A. (1910). The psychology of efficiency: An experimental study of the processes involved in the solution of mechanical puzzles and in the acquisition of skill in their manipulation. New York, NY: The Science Press.

Sandkühler, S., \& Bhattacharya, J. (2008). Deconstructing insight: EEG correlates of insightful problem solving. PLOS ONE, 3, e1459.
Schuck, N. W., Gaschler, R., Wenke, D., Heinzle, J., Frensch, P. A., Haynes, J. D., et al. (2015). Medial prefrontal cortex predicts internally driven strategy shifts. Neuron, 86, 331-340.

Selz, O. (1922). Zur Psychologie der produktiven Denkens und des Irrtums (On the psychology of productive thinking and of error). Bonn: Cohen.

Seyed-Allaei, S., Amati, D., \& Shallice, T. (2010). Internally driven strategy change. Thinking and Reasoning, 16, 308-331.

Shallice, T., \& Cooper, R. P. (2011). The organisation of mind. Oxford: Oxford University Press.

Shallice, T., \& Cooper, R. P. (2013). Is there a semantic system for abstract words? Frontiers in Human Neuroscience, 7, 175.

Sio, U. N., \& Ormerod, T. C. (2009). Does incubation enhance problem solving? A meta-analytic review. Psychological Bulletin, 135, 94-120.

Tomasi, D., \& Volkow, N. D. (2012). Gender differences in brain functional connectivity density. Human Brain Mapping, 33, 849-860.

Vartanian, O., \& Goel, V. (2005). Task constraints modulate activation in right ventral lateral prefrontal cortex. Neuroimage, 27, 927-933.

Woodworth, R. S., \& Schlosberg, H. (1955). Experimental psychology. New York: Henry Holt. 